\title{
Cycle et rôle du silicium d'origine végétale dans les écosystèmes forestiers tempérés
}

\author{
F. BARTOLI et B. SOUCHIER
}

Centre de Pédologie Biologique, C.N.R.S.,

17, rue Notre-Dame-des-Pauvres, 54500 Vandœuvre

\begin{abstract}
Résumé
La silice est à la fois constituant essentiel des minéraux des sols et de la composition minérale des végétaux. Elle est en effet intensément recyclée par la végétation où elle est stockée dans les tissus épidermiques des feuilles sous forme d'incrustations siliceuses appelées phytolithes.

L'étude de cette silice d'origine végétale permet de différencier les écosystèmes forestiers et les teneurs en silice foliaire donnent des informations physiologiques, pédogénétiques, voire sylvicoles et agronomiques, très intéressantes.

Ainsi, la teneur en silice foliaire est un bon indicateur de la transpiration de la plante, et par là, un élément de diagnostic pour utiliser certaines pratiques sylvicoles d'irrigation ou d'éclaircies.

D'autre part, la silice d'origine végétale (phytolithes) est un bon traceur de l'activité biologique des litières et de l'humus. Ainsi, les phytolithes s'accumulent dans les podzols, à activité biologique ralentie, où elles fixent dans les horizons spodiques $B_{h}$ une partie de l'azote facilement accessible à l'activité biologique.

A l'opposé, les phytolithes sont rapidement biodégradés en milieu biologiquement actif et le silicium rendu ainsi assimilable pour la végétation, permettrait d'augmenter la croissance des peuplements forestiers.
\end{abstract}

\section{Introduction}

La silice est à la fois constituant essentiel des minéraux des sols et de la composition minérale des végétaux. II n’est donc pas étonnant que son rôle dans la production agricole ait été depuis longtemps étudié et discuté.

Les agronomes tropicaux pensent actuellement que la silice a des effets bénéfiques sur les plantes, notamment les céréales. Ainsi, l'épandage de scories, résidus des aciéries (pratique très courante au Japon), de paille de riz, riche en silice(Madagascar) peut accroître les rendements en riz de plus de 20 p. 100 (Velly, 1974).

L'application de silice a également été favorable pour une large gamme de cultures comme la betterave, le tournesol, le maïs, la tomate, le tabac, l'ananas, la canne à sucre (Halais et Parish, 1963) et le jute (Khan et Roy, 1964).

En milieu tempéré, par contre, le rôle du silicium est plus mal connu des agronomes et des écologistes de la forêt. Aussi, nous nous sommes attachés à étudier le cycle biologique du silicium dans des écosystèmes forestiers tempérés.

Nous rendons compte ici des résultats essentiels de ce travail et de leurs implications dans des domaines variés : pédogenèse et paléobotanique d'une part, physiologie et productivité végétale d'autre part. 


\section{I. - Stations et méthodologie}

\section{1. - Stations}

Notre étude se limite à des stations de sol brun acide, sol podzolique et podzol sur roches gréseuses (tabl. 1). Des études pédogénétiques approfondies ont été menées

\section{TABLEAU 1}

Caractérisation des stations

Characterization of the stations

\begin{tabular}{|c|c|c|c|c|c|}
\hline Végétation & Sol & Type d'humus & Roche-mère & Lieu & Décrit par \\
\hline \multirow{2}{*}{$\begin{array}{l}\text { Hêtraie } \\
\text { Hêtraie-sapinière } \\
\text { à fétuque }\end{array}$} & Sol brun acide & Mull & Grès rhétien & Plaine lorraine & F. Toutain, 1974 \\
\hline & Sol brun acide & Mull acide & Grès vosgien & Bosses-Vosges & $\begin{array}{l}\text { B. Guillet et } \\
\text { J. Roviller, } 1974\end{array}$ \\
\hline $\begin{array}{l}\text { Hêtraie } \\
\text { Sapinière } \\
\text { Pineraie }\end{array}$ & $\begin{array}{l}\text { Sol podzolique } \\
\text { Sol podzolique } \\
\text { Podzol }\end{array}$ & $\begin{array}{l}\text { Moder } \\
\text { Moder } \\
\text { Mor }\end{array}$ & $\begin{array}{l}\text { Grès rhétien } \\
\text { Grès vosgien } \\
\text { Grès vosgien }\end{array}$ & $\begin{array}{l}\text { Plaine lorraine } \\
\text { Basses-Vosges } \\
\text { Basses-Vosges }\end{array}$ & $\begin{array}{l}\text { F. Toutain, } 1974 \\
\text { B. Guillet, } 1972 \\
\text { B. Guillet et } \\
\text { J. Rouiller, } 1974\end{array}$ \\
\hline
\end{tabular}

dans ces stations par plusieurs équipes du Centre de Pédologie biologiques qui poursuit aujourd'hui des recherches sur les équilibres sol-solution grâce à des dispositifs lysimétriques (Vedy, 1976).

\section{2. - Isolement et dosage des phytolithes}

Les méthodes d'isolement, de détermination des phytolithes et de dosage de la silice d'origine végétale présente dans les feuilles, les litières ou le sol sont décrites en détail dans un article antérieur (Bartoli et al., 1976).

\section{3. - Détermination d'un bilan approché du silicium}

A partir des teneurs en silicium des différents constituants des arbres (racines, troncs, rameaux, feuilles ou aiguilles : première, deuxième année et plus anciennes) et des plantes de la strate herbacée, nous avons pu calculer la quantité de silicium retenu chaque année dans la biomasse.

- Le silicium végétal restitué par la litière a été quantifié (dosage de Si et pesée des retombées annuelles).

- La masse de silicium absorbé chaque année par les racines représente la somme silicium retenu + silicium restitué.

- Dans le profil du sol, nous avons par ailleurs isolé la silice d'origine végétale. Nous avons ainsi pu établir la masse de phytolithes de la retombée annuelle $(P)$, de la biomasse $\left(P_{1}\right)$, des litières $\left(P_{2}\right)$, des humus et des horizons minéraux $\left(P_{3}\right) \cdot P_{1} / P$, 
$P_{2} / P, P_{3} / P$ représentent les temps de résidence moyens du silicium d'origine biologique à chaque niveau de l'écosystème végétation-sol.

Nous avons également quantifié la part du silicium d'origine minérale (fraction argiles, minéraux primaires, feldspaths et quartz, les autres silicates étant négligeables dans les sols étudiés).

- Dans les eaux, les teneurs en silicium ont été déterminées par absorption atomique après ultrafiltration (millipore $0,45 \mu$ ). Grâce aux dispositifs lysimétriques installés dans les stations, des mesures saisonnières ont permis d'établir le bilan net du silicium exporté hors du profil.

\section{II. - Cycle biologique du silicium}

\section{A. - Absorption et accumulation dans les plantes : mécanismes et facteurs}

\section{1. - Rappel succinct des mécanismes.}

Les racines extrayant le silicium du sol jouent un rôle actif et déterminant dans l'accumulation de la silice végétale (Mitsui et Takatoh, 1963 ; Okuda et Takahashi, 1964).

Par la suite, le silicium suivra de manière passive le flux de sève auquel il s'associe (Sangster, 1970 ; Robert et al., 1973).

Sans entrer dans le détail des mécanismes de l'accumulation du silicium dans les plantes, rappelons que l'anion silicaté soluble, parvenu au niveau des vacuoles des cellules épidermiques des feuilles, s'associe avec des protéines pour se fixer finalement sur une trame polysaccharidique (callose ou cellulose) (Sangster, 1970 ; Robert et al., 1973).

Par dessiccation progressive liée à la transpiration, la silice initialement soluble évolue à l'état de gel, puis d'opale (silice hydratée amorphe) qui constitue les phytolithes, incrustations silicieuses épousant le contour des cellules épidermiques des feuilles (Yoshida et al., 1962, 1969).

\section{2. - Le facteur essentiel du cycle biologique du silicium : le facteur plante.}

Le type de plante intervient essentiellement par la capacité spécifique d'absorption racinaire, elle-même reliée à la capacité de transpiration (Bartoli et Beaucire, 1976). Ainsi, la teneur en silicium d'une plante est essentiellement caractéristique de l'espèce $\left({ }^{1}\right)$. Il y a par exemple dix fois plus de silicium dans les aiguilles de sapin que dans celle de pin (Bartoli et Beaucire, 1976), et vingt fois plus dans les feuilles de graminées que dans celles de légumineuses (Jones et Handreck, 1967).

Comme nous venons de le dire, la vitesse d'absorption du silicium des racines jusqu'aux feuilles est voisine de celle du flux de sève : de l'ordre de quelques heures (Mitsui et Takatoh, 1963 ; Shim et Yoo, 1967). Cette vitesse rapide rend ainsi très

( $\left.{ }^{1}\right)$ Voir en annexe une revue bibliographique des teneurs en silice de nombreuses espèces d'écosystèmes forestiers tempérés. 
difficile le stockage du silicium sur ses lieux de passage (racines, bois, écorce). Le silicium migre ainsi rapidement vers les feuilles où il s'accumule là où l'eau de la sève s'évapore le plus : épiderme, apex, stomates (Baba, 1955 ; Yoshida et al., 1962 ; Parry et Smithson, 1964 ; Jones et Handreck, 1967 ; Laroche ,1967 ; Soni et al., 1972).

L'étroite relation qui existe donc entre le flux de sève et la quantité de silicium foliaire laisse prévoir l'action de facteurs secondaires qui peuvent modifier l'intensité de la transpiration (âge des organes foliaires et du peuplement) ou les conditions de l'assimilation (sol).

3. - Les facteurs secondaires : âge, climat, sol.

- Age des organes foliaires.

- Pour les feuilles, la teneur en silicium (comme la plupart des éléments minéraux) augmente régulièrement avec la saison de végétation et peut être couramment multipliée par 5 entre le printemps ef l'automne (fig. 1, Bartoli et Beaucire, 1976).
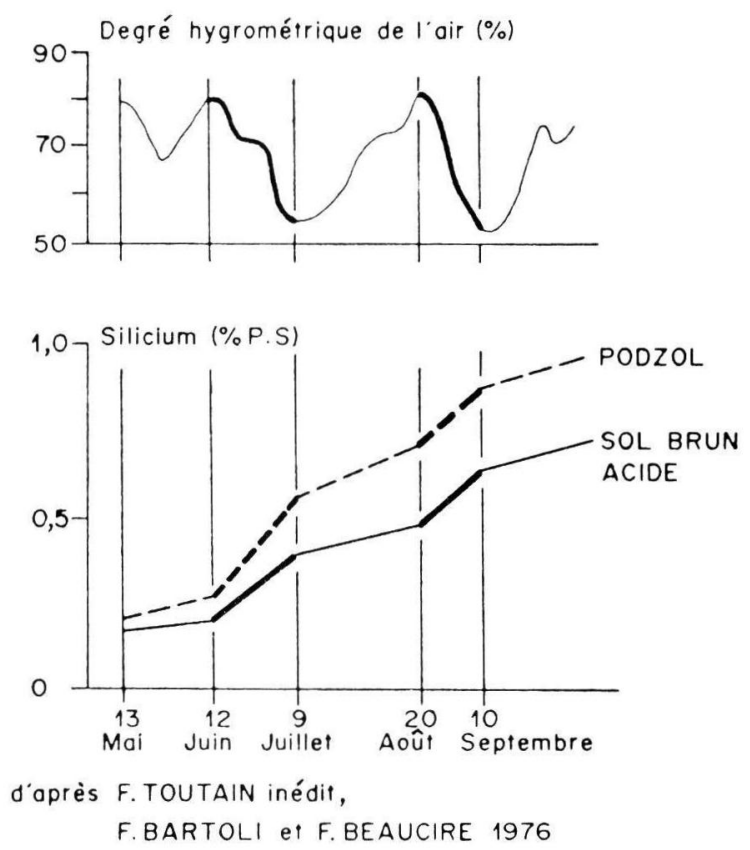

FIG. 1. - Accumulation du silicium dans les feuilles de Hêtre (Fagus silvatica).

Silicon accumulation in the beech leaves (Fagus silvatica).

- L'accumulation du silicium dans les aiguilles de résineux est continue jusqu'à leur chute, mais elle se ralentit beaucoup en fin de troisième année (Fig. 2, tabl. 2).

- Age du peuplement.

L'accumulation de silicium dans les feuilles est d'autant plus importante que le peuplement est plus jeune et le flux de sève plus intense (tabl. 3 ). 

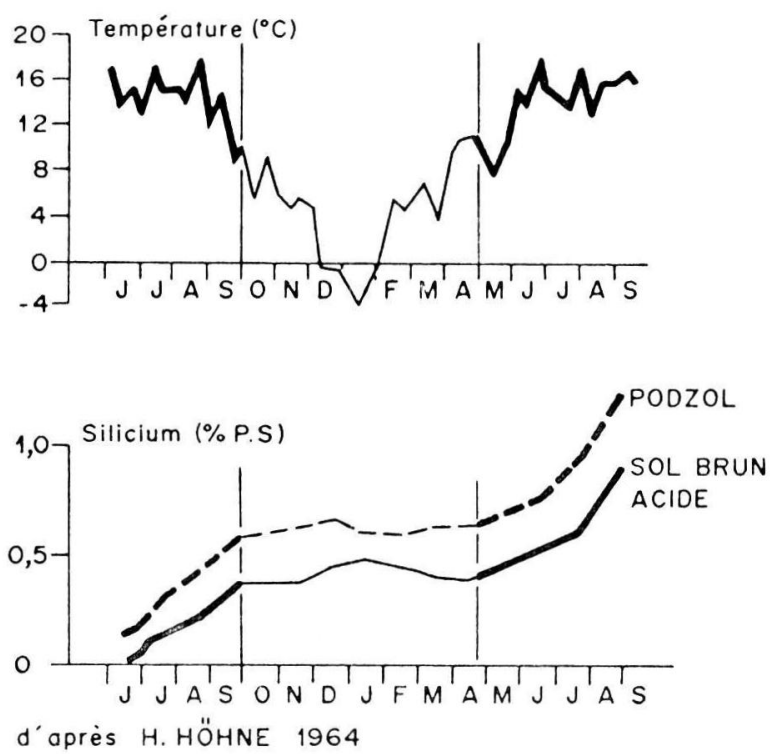

FIG. 2. - Accumulation du silicium dans les aiguilles (0 à 1 an 1/2) d'Epicéa (Picea exelsa, peuplement de 15 ans).

Silicon accumulation in the Norway Spruce 0 to 1 and $a$ half year old needles (Picea exelsa, 15 years old plantation).

TABLEAU 2

Teneur en silicium en fonction de l'âge des aiguilles de résineux (d'après Fiedler et Höhne, 1965) Silicon content in relationship with the resinous needles age (after Fiedler and Höhne, 1965)

\begin{tabular}{|c|c|c|}
\hline & Epicea & Douglas \\
\hline Age de l'aiguille & $\begin{array}{l}\text { Teneur en Si } \\
\text { p. } 100 \text { P. S. }\end{array}$ & $\begin{array}{l}\text { Teneur en Si } \\
\text { p. } 100 \text { P. S. }\end{array}$ \\
\hline $\begin{array}{l}1 \text { an } \ldots \ldots \ldots \ldots \\
2 \text { ans } \ldots \ldots \ldots \ldots \ldots \\
3 \text { ans } \ldots \ldots \ldots \ldots \ldots \\
4 \text { ans } \ldots \ldots \ldots \ldots\end{array}$ & $\begin{array}{l}0,73 \\
1,68 \\
2,20 \\
2,35\end{array}$ & $\begin{array}{l}0,43 \\
0,94 \\
1,36 \\
1,71\end{array}$ \\
\hline
\end{tabular}

TABLEAU 3

Influence de l'âge du peuplement sur la teneur en silicium foliaire (d'après Pavlov, 1972) The role of the plantation age on the leave silicon content (after Pavlov, 1972)

\begin{tabular}{lccc}
\hline & $\begin{array}{c}\text { Age du } \\
\text { peuplement } \\
\text { (années) }\end{array}$ & $\begin{array}{c}\text { Teneur en } \\
\text { silicium } \\
\text { p. 100 P. S. }\end{array}$ & $\begin{array}{c}\text { Biomasse } \\
\text { silicium } \\
\mathrm{kg} / \mathrm{ha}\end{array}$ \\
\hline Hêtre & 60 & 0,65 & 100 \\
& 80 & 0,51 & 86 \\
Sapin & 120 & 0,46 & 100 \\
& 20 & 1,13 & 82 \\
\hline \hline
\end{tabular}


- Le climat.

Le climat intervient sur la transpiration, ef donc sur la concentration en silicium dans la biomasse : l'accumulation de silicium dans les aiguilles ou les feuilles s'accélère pendant les périodes sèches et chaudes d'évapotranspiration intense, et de fort déficit hydrique atmosphérique (fig. 1 et 2). De même, les feuilles ou aiguilles hautes, situées dans les zones d'éclairement maximales, sont généralement les plus riches en silicium (tabl. 4, d'après Bartoli et Beaucire, 1976).

\section{TABLEAU 4}

Relation entre la silicification foliaire et la transpiration liée à l'éclairement (arbres poussant sur le même podzol du Bassin Parisien)

Relationship between the leave silicification and the sap flow intensity correlated with the light intensity (the trees grow on the same spodosol near Paris)

\begin{tabular}{lcccc}
\hline \hline & $\begin{array}{c}\text { Position des } \\
\text { feuilles/sol } \\
(\mathrm{m})\end{array}$ & $\begin{array}{c}\text { Silicium foliaire } \\
(\mathrm{mg} \text { par arbre }) \\
\mathrm{Si}\end{array}$ & $\begin{array}{c}\text { Surface foliaire } \\
\left(\mathrm{m}^{2} \text { par arbre }\right) \\
\text { SF }\end{array}$ & $\begin{array}{c}\text { Coefficient de } \\
\text { corrélation } \mathrm{r} \\
\mathrm{Si}-\mathrm{SF}\end{array}$ \\
\hline Feuilles de Bouleau & 1 & 105 & 5,0 & \\
Aiguilles de Pin & 3 & 153 & 6,7 & 0,962 \\
& 6 & 309 & 8,5 & \\
& 1 & 105 & 4,0 & 0,948 \\
\hline
\end{tabular}

- Le sol.

La teneur en silicium d'espèces écologiquement très plastiques, comme le Hêtre ou le Sapin, peut varier avec le type de sol. Il apparaît que le cycle biologique du silicium est, pour une espèce donnée, d'autant plus efficace que le milieu est plus acide : ainsi, pour le Hêtre (fig. 1), le Sapin (Bartoli et Beaucire, 1976) et l'Epicéa (fig. 2), l'assimilation du silicium paraît maximale dans les sols podzolisés où la solution du sol semble également plus riche en silicium (Bartoli et Beaucire, 1976).

Le sol pourrait intervenir de façon très complexe sur la vitesse d'assimilation du silicium ; la forme de la silice, monomère ou complexée organique dans le sol serait en cause (Holzapfel, 1953 ; Holzapfel et Engel, 1956) ainsi que le $\mathrm{pH}$ de la sève, lui-même lié à la teneur du sol en certains éléments nutritifs (Delmas, 1960).

\section{B. - Bilan global du silicium ef évolution dans l'écosystème}

\section{1. - Retombées annuelles et rapport silicium biologique/silicium minéral.}

La retombée annuelle de silice d'origine végétale est généralement deux fois plus grande dans les écosystèmes feuillus que dans les écosystèmes résineux (fig. 3 ).

Dans les sols acides aérés étudiés, le rapport silicium d'origine végétale/silicium d’origine minérale est toujours extrêmement faible : 1/1 000 à 1/5 000 (tabl. 5). 
TABLEAU 5

Bilan du silicium (en $t / h a$ ) des phytolithes et des minéraux d'un sol brun acide (hêtraie-sapinière) et d'un podzol (pineraie) pour $50 \mathrm{~cm}$ de profondeur de sol

Silicon balance (in $t / h a$ ) of the phytoliths and the minerals of the acid brown soil (beech-fir plantation) and the spodosol (pinewood) for $50 \mathrm{~cm}$ soil depth

\begin{tabular}{lcccc}
\hline \hline & Phytolithes & Argiles & Feldspaths & Quartz \\
\hline Sol brun acide... & 0,5 & 50 & 172 & 2145 \\
Podzol $\ldots \ldots \ldots$ & $2,1 *$ & 41 & 92 & 2100 \\
\hline $\begin{array}{l}\text { * } 1,35 \text { t/ha phytolithes de Pin }+0,75 \text { t/ha phytolithes anciennes de Callune } \\
\text { (Bartoli et Guillet, 1977). }\end{array}$
\end{tabular}

2. - Accumulation des phytolithes dans les humus ef les sols (facteurs de biodégradation).

- Les caractéristiques physico-chimiques des phytolithes de chaque type de végétal déterminent fondamentalement la vitesse de biodégradation. Ainsi, la rigidité des phytolithes de résineux explique leur accumulation relative dans les sols : le temps de résidence moyen des phytolithes de Sapin dans le sol (litières + horizons) est de 80 ans dans la sapinière sur le sol podzolique, à activité biologique moyenne (tabl. 6), de 60 ans dans la hêtraie-sapinière à forte activité biologique (Bartoli et Selmi, 1977).

A l'opposé, la fragilité des phytolithes de feuillus (Bartoli et Selmi, 1977) favorise leur biodégradation. Les phytolithes de Hêtre disparaissent ainsi plus vite dans les sols podzoliques (temps de résidence moyen dans le sol : 27 ans) que celles de Sapin (80 ans) ou de Pin (300 ans) (fig. 3, tabl. 6).

\section{TABLEAU 6}

Devenir du silicium d'origine végétale dans les plantes, les litières et les sols

The evolution of biogenetic silicon in the plants, the litters and the soils

\begin{tabular}{|c|c|c|c|}
\hline \multirow{2}{*}{$\begin{array}{l}\text { Peuplement et } \\
\text { type de sol }\end{array}$} & \multicolumn{3}{|c|}{ Temps de résidence moyen des phytolithes (en années) } \\
\hline & $\begin{array}{l}\text { Dans les plantes } \\
\text { (feuilles ou aiguilles) }\end{array}$ & Dans les litières & $\begin{array}{l}\text { Dans le sol } \\
\text { (horizons organo-minéraux) }\end{array}$ \\
\hline $\begin{array}{l}\text { Hêtraie } \\
\text { Sol brun }\end{array}$ & 0,5 & 2,0 & 1 \\
\hline $\begin{array}{l}\text { Hêtraie-Sapinière } \\
\text { Sol brun acide }\end{array}$ & 1,1 & 4,0 & 25 \\
\hline $\begin{array}{l}\text { Hêtraie } \\
\text { Sol podzolique }\end{array}$ & 0,5 & 4,0 & 25 \\
\hline $\begin{array}{l}\text { Sapinière } \\
\text { Sol podzolique }\end{array}$ & 2,4 & 9,5 & 70 \\
\hline $\begin{array}{l}\text { Pineraie } \\
\text { Podzol }\end{array}$ & 2,4 & 10,0 & $300 *$ \\
\hline
\end{tabular}

\footnotetext{
* Pin (Bartoli et Guillet, 1977).
} 


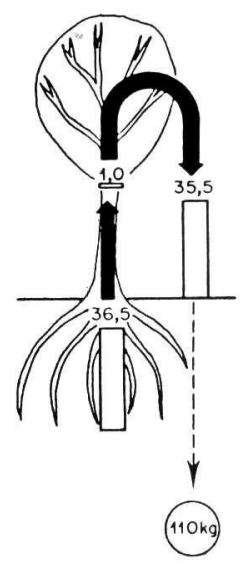

HÊTRAIE SUR SOL BRUN ACIDE
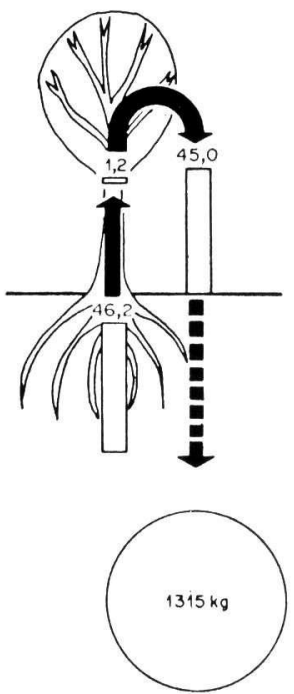

HÊTRAIE SUR SOL PODZOLIQUE

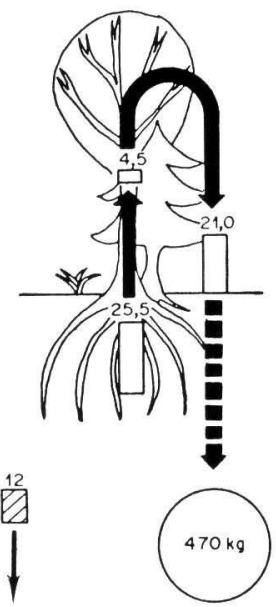

HÊTRAIE-SAPINIERE SUR SOL BRUN ACIDE
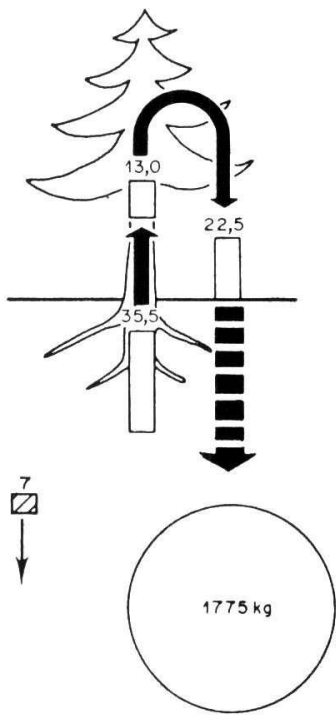

SAPINIÉRE SUR SOL PODZOLIQUE

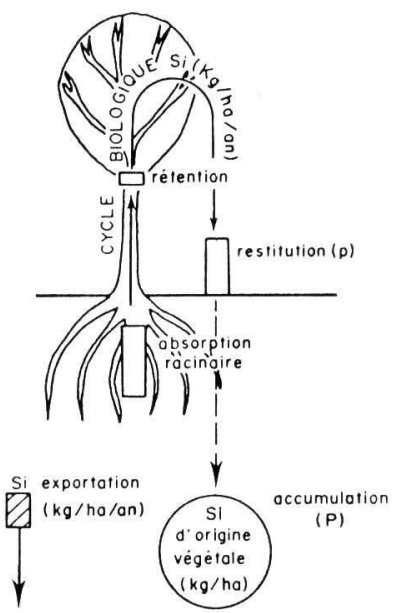

LEGENDE

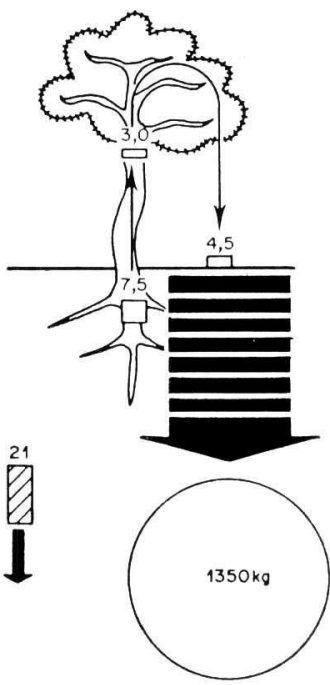

PINERAIE SUR PODZOL

FIG. 3. - Cycle biologique du silicium en milieu acide aéré (grès, Lorraine et Vosges). L'épaisseur des flèches descendantes en tireté est proportionnelle au temps de résidence moyen des phytolithes dans les sols, exprimé par le rapport $P / P(P=$ masse de phytolithes accumulées dans le sol, représentée par un cercle, $p=$ masse de phytolithes restituées chaque année au sol par la litière, représentée par un rectangle).

Biological cycle of silicon in the aerated acid soils (sandstone, Eastern France). The thickness of the dash descending lines is proportional to the average residence time of the phytoliths in the soils, expressed by the $P / P$ ratio $(P=$ soil phytoliths quantity represented by a circle, $P=$ annual litter phytoliths represented by a rectangle). 
- L'activité biologique du milieu sol influe aussi sur le temps de résidence des phytolithes dans le sol. Ainsi, la faible activité biologique des podzols explique une accumulation relative des phytolithes dans le sol. A l'opposé, l'activité biologique intense des sols bruns induit une très forte solubilisation du silicium d'origine végétale (fig. 3 et tabl. 6) (Bartoli et Selmi, 1977).

L'exemple des hêtraies sur sol brun acide et sol podzolique est très probant à cet égard : le temps de résidence moyen des phytolithes dans les litières est de 4 ans dans le sol podzolique et de 2 ans dans le sol brun. Mais cette divergence s'accentue brutalement dans le sol : le temps de résidence moyen des phytolithes dans le sol (horizons organo-minéraux) est de 25 ans dans le sol podzolique à activité biologique moyenne et seulement d'un an dans le sol brun à très forte activité biologique (fig. 3 , tabl. 6 ; Bartoli et Selmi, 1977).

Le temps de résidence des phytolithes dans les sols est ainsi un bon critère de l'activité biologique dans les humus.

II faut de plus noter que les deux facteurs : activité biologique et caractéristiques de phytholithes ne sont sans doute pas indépendants. La résistance des phytolithes de résineux, charpente minérale des aiguilles, tend à freiner la biodégradation des litières, ce qui n'est pas du tout le cas pour les fragiles incrustations siliceuses des écosystèmes feuillus.

- La capacité d'absorption du silicium par la plante (Bartoli et Beaucire, 1976) intervient grandement dans le cycle biogéochimique du silicium : il y a d'autant moins de silicium exporté hors du profil que la végétation absorbe plus de silicium. Il y a par exemple, opposition (fig. 3) entre une pineraie à faible capacité racinaire d'absorption du silicium $(7,5 \mathrm{~kg} / \mathrm{ha} / \mathrm{an})$ et une sapinière à forte capacité d'absorption $(35,5 \mathrm{~kg} / \mathrm{ha} / \mathrm{an})$ : dans le premier cas, le silicium de la solution du sol, peu prélevé par la plante, est exporté hors du profil trois fois plus intensément ( $21 \mathrm{~kg} \mathrm{Si} / \mathrm{ha} / \mathrm{an}$ ) que dansla sapinière (perte annuelle de $7 \mathrm{~kg} \mathrm{Si} / \mathrm{ha}$ ). Les processus d'absorption du silicium hydrosoluble sur des fractions minérales interviennent toutefois pour nuancer cette interprétation.

3. - Modes de migration du silicium d'origine végétale. Significations pédogénétiques.

On peut, à partir des bilans établis dans les précédents paragraphes, pressentir l'importance de l'équilibre silicium biologique-silicium minéral dans les processus d'altération et d'absorption.

Le silicium d’origine végétale peut migrer sous deux formes :

- A l'état soluble ou pseudosoluble.

Le silicium serait peu polymérisé et pourrait parfois être associé à des macromolécules organiques. C'est en tout cas ce qui semble ressortir d'expériences d'altérations biologiques en flux semi-continue, de mesures colorimétriques et d'encombrement moléculaire. Une grande quantité de ce silicium hydrosoluble d'origine végétale s'insolubilise dans l'horizon humifère des sols bruns (Bartoli et al., 1977).

Dans les podzols, au contraire, une fraction soluble de silice biologique vient précipiter dans l'horizon $B_{\mathrm{S}}$ marqué effectivement par une accumulation de silice amorphe plus ou moins associée à l'aluminium (Brydon et Shimoda, 1972) et facilement extraite par le réactif de Tamm (tampon oxalique, $\mathrm{pH} 3,5$ ). Il est à noter que cette accumulation 
de silice amorphe procède à la fois du cycle biologique de la silice, mais plus encore de la destruction des silico-aluminates dans les horizons de surface des podzols.

\section{- A l'état particulaire.}

Sous forme de phytolithes plus ou moins débarrassées de leur gangue organique et plus ou moins lestées par de fines particules minérales (argiles micacées inférieures à $0,5 \mu$ ) (Bartoli et Guillet, 1977). Cette migration s'effectue essentiellement dans les podzols où elle aboutit à des diagrammes de répartition des phytolithes suivant la profondeur. Les indications données par ces diagrammes viennent compléter celle des spectres polliniques. Les phytolithes, généralement plus fragiles que les pollens retracent une histoire de la végétation plus courte, limitée à l'environnement stationnel immédiat, et à quelques espèces sélectivement conservées, tandis que les pollens enregistrent une histoire plus longue et plus régionale de la flore (Bartoli et Guillet, 1977).

Au cours de cette migration, les phytolithes peuvent intervenir comme source de silicium et microsites d'agradation siliceuse d'argiles fines fixées sur leurs surfaces (Bartoli, 1977). Elles jouent aussi un rôle biochimique non négligeable en fixant préférentiellement de l'azołe aminé facilement disponible pour l'activité biologique (Bartoli, et al., 1977).

\section{III. - Rôles physiologiques et nutritionnels du silicium : conséquences agronomiques et sylvicoles}

Le cycle biologique du silicium paraît intervenir dans les mécanismes de l'assimilation minérale par les végétaux et avoir aussi un rôle complexe sur la productivité des écosystèmes forestiers.

Nous évoquerons ici rapidement, à partir de quelques données bibliographiques, les actions bénéfiques du silicium sur la croissance végétale.

\section{1. - Silicium et photosynthèse}

Le rôle actif du silicium vis-à-vis de la photosynthèse a été démontré. En effet, le silicium, par sa rigidité, permet une meilleure disposition des feuilles vis-à-vis du rayonnement solaire et permet donc d'augmenter la capacité de photosynthèse de la plante (Iwata et Baba, 1962 ; Yoshida et al., 1969 ; Cock et Yoshida, 1970).

\section{2. - Silicium et réserves en eau}

L'accumulation de silice dans les stomates provoque la formation d'une double cuticule siliceuse qui, en réduisant la transpiration, diminue les besoins en eau des plantes. Ainsi, Boresch et Sachse (1928) ont montré qu'un apport de silice facilement assimilable (silicate de sodium) diminuait de près de 35 p. 100 les besoins en eau d'une avoine.

3. - Silicium, résistance au froid et aux maladies cryptogamiques

De nombreux chercheurs ont montré qu'un apport de silice accroît la résistance des céréales aux maladies et aux parasites (Ishibashi et Akiyama, 1960 ; Lanning 
et Lindo, 1961 ; Yoshida ef al., 1962 ; Adlakha, 1964), et remplacerait avantageusement les insecticides souvent nocifs vis-à-vis des chaînes alimentaires. L'exemple d'une culture de riz pluvial sur sol brun-rouge ferrallitique malgache (Velly, 1974) est significatif à cet égard (tabl. 7).

\section{TABLEAU 7}

Rôle comparé de la silice et des insecticides (d'après Velly, 1974)

Compared role of silica and of insecticides (after Velly, 1974)

\begin{tabular}{|c|c|}
\hline & Rendements $(\mathrm{kg} / \mathrm{ha})$ \\
\hline 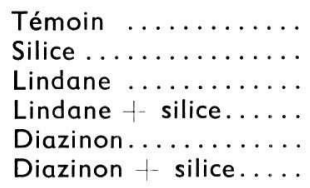 & $\begin{array}{ll}2 & 617 \\
3 & 084 \\
2 & 949 \\
3 & 345 \\
3 & 161 \\
3 & 372\end{array}$ \\
\hline
\end{tabular}

4. - Silicium et assimilabilité des éléments majeurs

L'apport de silicium permet une meilleure assimilabilité du potassium (Velly, 1974) et surtout du phosphore (Raupach et Piper, 1960 ; Ganssmann, 1962, Du Plessis et Du Burger, 1966 ; Bastisse, 1967).

En effet, le silicium s'échange avec le phosphore dans le complexe absorbant des sols (Kafkafi et Bar Yosef, 1969), lui permettant d'être ainsi plus assimilable par les plantes.

D'autre part, on observe que la solubilité du phosphore des scories basiques augmente avec la teneur en silicium des scories (Gericke, 1962).

\section{5. - Silicium et oligoéléments}

L'accumulation du silicium dans les feuilles freine celle des oligo-éléments (Cu, $\mathrm{Zn}, \mathrm{Fe}, \mathrm{Mn}$...) (Lewin et Reimann, 1969). Ainsi, le silicium lèverait la toxicité d'un élément comme le manganèse, non seulement en diminuant la teneur en manganèse absorbé, mais surtout en provoquant une micro-redistribution du manganèse dans les feuilles (effet de dilution) (Vlamis et Williams, 1967 ; Bowen, 1972).

\section{Conclusions}

Dans le cycle biologique des éléments minéraux, le silicium est, avec les cations alcalino-terreux, préférentiellement absorbé par les écosystèmes forestiers. Plusieurs aspects du cycle biologique du silicium ont retenu notre attention :

- L'intensité de l'accumulation du silicium dans la biomasse :

- La capacité d'absorption dépend essentiellement de l'espèce et de la composition de l'association floristique ; ainsi les graminées sont généralement plus riches que les autres espèces herbacées, notamment les espèces acidiphiles, de la couverture végétale. 
- La teneur en silicium foliaire augmente régulièrement avec la saison de végétation.

- Le facteur climat, dans la mesure où il intervient sur l'intensité de la transpiration, peut activer, notamment en période estivale, à fort déficit hydrique, l'absorption et l'accumulation du silicium.

- Dans la mesure où le flux de sève tend à diminuer avec l'âge des arbres, l'accumulation du silicium dans les peuplements forestiers tend vers une valeur limite, plus rapidement atteinte pour les feuillus que pour les résineux.

- L'influence du type de sol n'est que très secondaire sur l'assimilation du silicium qui semble favorisée cependant par l'acidité du milieu.

- L'évolution dans les humus et les sols de la silice d'origine végétale - les phytolithes - diffère très nettement en fonction du type de sol.

Le cycle biologique de la silice a donc une signification pédogénétique importante qui, dans les sols acides aérés étudiés, différencie la brunification et la podzolisation :

- Dans les sols bruns à activité biologique élevée, caractérisant le climax de la hêtraie-sapinière à mull, les phytolithes qui constituent de fines incrustations siliceuses dans la matière organique fraîche sont rapidement dissoutes et subissent un renouvellement rapide.

Il est difficile de savoir quel rôle actif peut être attribué à cette silice rapidement mobilisée à l'état soluble ou pseudosoluble : il nous est toutefois permis d'imaginer ses effets physiologiques bénéfiques sur la croissance végétale par une augmentation de la capacité photosynthétique, une meilleure régulation en eau, une meilleure assimilabilité en éléments nutritifs, une résistance accrue au froid, aux maladies et aux toxicités chimiques.

Le bilan du silicium ne fait ressortir que des pertes faibles dans l'écosystème à mull actif, ce qui pourrait signifier que le silicium d'origine végétale est fixé par le complexe argilo-humique, la microflore, et réabsorbé rapidement par le système racinaire. Quoi qu'il en soit, le recyclage par la végétation freine la perte de silicium du bilan géochimique général.

- Dans les sols podzoliques et podzols à moder ou mor, les peuplements résineux favoriseraient au contraire la conservation, dans l'humus et le sol, d'une fraction phytolithique importante, malgré un cycle biologique beaucoup moins intense.

La migration des phytolithes, à l'image de celle des pollens, permet de retracer, au moins partiellement, l'histoire de la végétation de ces milieux podzolisés. Le rôle pédogénétique de cette fraction phytolithique à dissolution lente est encore mal établi. On peut cependant concevoir que, dans ces milieux, la structure moins labile des phytolithes tend à retarder la décomposition des humus et puisse avoir en même temps un rôle de micro-sources de silicium susceptibles d'intervenir dans l'équilibre thermodynamique des fractions fines argileuses riches en silice.

Enfin, les phytolithes s'enrichissent, au cours de leur migration, en azote facilement disponible pour l'activité biologique. Ceci serait dô à leur réactivité élevée visà-vis des protéines.

Enfin, l'intensité du cycle biologique de la silice, en relation avec le type de station (association floristique-humus-sol) peut avoir des incidences sur l'équilibre physiolo- 
gique du peuplement forestier et sa productivité : il est vraisemblable que l'effet bénéfique de certaines interventions sylvicoles (mélanges d'essences, éclaircies, apport d'engrais silicatés, etc...) soit au moins partiellement lié à l'activation du cycle biologique de la silice.

Reçu pour publication en décembre 1977.

\title{
Summary
}

Cycle and role of biogenetic silica in temperate forest ecosystems

In the plant silicon accumulation process, the plant type plays the main role, determined by the root's capacity to absorb more or less silicon ; leaf age, plantation age, climate and soil are only secondary factors.

In the humus and in the soils, the evolution of biogenetic silica has a pedogenetic signification which, in this study, differenciates the browning process and the podzolising one. Thus in « beech-fir plantation-brown soil with mull » ecosystems, biogenetic silica, in the form of brittle siliceous incrustations, is quickly dissolved; the intense biological cycle of silicon slows down the loss of silicon in the general geochemical balance.

On the contrary, in podzols with moder or mor, resineous plantations should favorize the conservation in the humus and in the soil, of biogenetic silica in spite of a weaker biological cycle.

Thus the study of biogenetic silica in podzols gives us interesting paleobotanical information.

Finally, the beneficial effects of some forestry interventions (forest species mixture, clearing, etc...) should at least partially be due to activation of the biological cycle of silicon.

\author{
Annexe \\ Teneurs en silice $\left(\mathrm{SiO}_{2}\right.$ en p. 100 P. S.) \\ d'espèces végétales d'écosystèmes forestiers tempérés
}

Espèces du mull eutrophe ou du mull calcique.

Mercurialis perennis : 0,2 p. $100 \mathrm{SiO}_{2}$ (P. S.) ; Urtica dioica : 1,1 à 4,7 p. 100 ; Senecio fuchsii : 0,5 à 1,5 p. 100 ; Brachypodium sylvaticum : 5,6 à 8,4 p. 100 (Höhne, 1963).

Espèces du mull mésotrophe.

Festuca sylvatica : 0,7 à 1,1 p. $100 \mathrm{SiO}_{2}$ (P. S.) (Bartoli et Beaucire, 1976 ; Melica uniflora : 2,3 à 4,1 p. 100 ; Agrostis tenuis : 2,9 à 3,0 p. 100 ; Poa chaixii : 0,7 à 0,9 p. 100 (Höhne, 1963).

Espèces hygrophiles.

Dryopteris filix-mas : 0,05 à 0,3 p. $100 \mathrm{SiO}_{2}$ (P. S.) (Höhne, 1963) ; Athyrium filix femina : 2,0 à 4,1 p. 100 (Höhne, 1963), 6,3 p. 100 (Kutuzova, 1968) ; Pteridium aquilinum : 1,1 à 1,9 p. 100 (Höhne, 1963) ; Molinia coerulea : 0,9 à 3,1 p. 100 (Höhne, 1963 ; Twiss et al., 1969).

Espèces du moder.

Deschampsia flexuosa : 0,4 à 2,0 p. $100 \mathrm{SiO}_{2}$ (P. S.) ; Deschampsia coespitosa : 2,5 à 4,1 p. 100 ; Luzula luzoloides : 0,4 à 1,1 p. 100 (Höhne, 1963) ; Calamagrostis epigeios : 4,0 à 6,3 p. 100 (Twiss et al., 1969). 


\section{Espèces du mor.}

Calluna vulgaris : 0,3 à 1,7 p. $100 \mathrm{SiO}_{2}$ (P. S.) (Höhne, 1963), 0,4 à 0,6 p. 100 (Bartoli et Beaucire, 1976) ; Vaccinium myrtillus : 0,1 à 0,3 p. 100 (Höhne, 1963 ; Bartoli et Beaucire, 1976).

\section{Espèces ubiquistes.}

Rubus idaeus : 0,02 à 0,3 p. $100 \mathrm{SiO}_{2}$ (P.S.) ; Melampyrum pratense : 1,3 à 1,4 p. 100 ; Calamagrostis villosa : 3,3 à 7,4 p. 100 (Höhne, 1963).

Feuillus.

Tremble : 1,2 p. $100 \mathrm{SiO}_{2}$ (P. S.) (Kutuzova, 1968) ; Bouleau : 0,3 à 0,4 p. 100 (Ovington, 1955 ; Kutuzova, 1968) ; Hêtre (Fagus sylvatica et Fagus grandifolia) : 0,5 à 2,4 p. 100 (Fiedler et Höhne, 1965 ; Wilding et Drees, 1971 ; Pavlov, 1972 ; Geis, 1973 ; Bartoli et Beaucire, 1976) ; Chêne (6 espèces) : 0,1 à 0,9 p. 100 (Ovington, 1955 ; Fiedler et Höhne, 1965 ; Verma et Rust, 1969 ; Geis, 1973 ; Wilding et Drees, 1974 ; Bartoli et Beaucire, 1976) ; Charme : 0,2 à 0,3 p. 100 (Fiedler et Höhne, 1965 ; Geis, 1973) ; Orme (trois espèces) : 2,1 à 3,6 p. 100 (Geis, 1973) ; Erable (deux espèces) : 0,3 à 1,0 p. 100 (Wilding et Drees, 1971 ; Geis, 1973).

\section{Résineux.}

Sapin : 0,8 à 1,4 p. $100 \mathrm{SiO}_{2}$ (P. S.) (Fiedler et Höhne, 1965, Kutuzova, 1968 ; Bartoli et Beaucire, 1976), 1,5 à 2,5 p. 100 (Pavlov, 1972) ; Epicéa : 0,3 à 3,7 p. 100 (Höhne, 1964 ; Fiedler et Höhne, 1965) ; Pin sylvestre : 0,05 à 0,1 p. 100 (Ovington, 1955 ; Bartoli et Beaucire, 1976) ; Mélèze : 0,9 à 1,0 p. 100 (Ovington, 1955).

\section{Références bibliographiques}

ADLAKHA P. A., 1964. Studies of the various factors responsible for resistance to top borer in the different varieties of sugarcane. Indian J. Sugarcane Res. Dev., 8 (3), 343-344.

BABA I., 1955. Translocation of absorbed silica in plants and its accumulation in the epidermal cells of leaf blades in relation to transpiration. Nippon Sakumotsu Gakkai Kiji, 24 (1), $29-33$ (résumé anglais, en japonais).

BARTOLI F., BEAUCIRE F., 1976. Accumulation du silicium dans les plantes vivantes en milieux pédogénétiques tempérés aérés. C. R. Acad. Sc. Paris, 282D, 1947-1950.

BARTOLI F., JEANSON Pierrette, DOIRISSE M., 1976. Isolement et caractérisation de la silice biologique (phytolithes) présente dans les feuilles, les litières et les sols en milieu aéré acide (Vosges gréseuses). Note technique $n^{\circ} 21$, C.P.B., 12 p.

BARTOLI F., GUILLET B., 1977. Etude comparée des diagrammes phytolitiques et polliniques d'un podzol des Vosges gréseuses. C. R. Acad. Sc. Paris, 284D, 353-356.

BARTOLI F., SELMI M., 1977. Sur l'évolution du silicium végétal en milieux pédogénétiques aérés acides. C. R, Acad. Sc. Paris, 284D, 279-282.

BARTOLI F., 1977. Biogenic silica as a major factor on pedogenetic migration process in aerated acid soils (East of France). Symposium Zvolen (Tchécoslovaquie), 32-41.

BARTOLI F., JOCTEUR MONROZIER Lucile, BELGY Marie-José, 1977. Etude comparée des fractions organo-minérales phytolithes et argiles d'un podzol des Vosges gréseuses. C. R. Acad. Sc. Paris, sous presse.

BARTOLI F., BOUDOU J. P., BELGY Marie-José, VEDY J. Cl., 1977. Formes et évolution des formes hydrosolubles du silicium des eaux libres et des eaux liées dans un sol brun et dans un podzol sur grès. En préparation.

BORESCH K., SACHSE J., 1928. Zur Frage nach der Bedeutung der Kieselsaüre in der Ernährung des Hafers. Festschrift zum 70 - Geburtstag von Stoklasa, Berlin, 167-176.

BOWEN J. E., 1972. Manganese-silicon interaction and its effect on growth of Sudan grass. Plant and Soil, 37, 577-588. 
COCK J. H., YOSHIDA S., 1970. An assessment of the effects of silicate application on rice by a simulation method. Soil Sc. Plant Nutr., 16 (5), 212-214.

DELMAS L., 1960. Relation entre le pH, la silice totale et la silice mobile des épis de blé. C. $R$. Acad. Sc. Paris, 251D, 2402-2404.

DU PLESSIS S. F., DU BURGER R. T., 1966. Silicate-phosphate interrelationships in soils. S. Afr. J. Agric. Sci., 9, 525-534.

FIEDLER H. S., HOHNE H., 1965. Vorkommen und Gehalt der Makronährstoffe in Waldbaümen. Wissenschaft. Zeitsch. der Technisc., Univ. Dresden, 14, 989-999.

BRYDON J. E., SHIMODA S., 1972. Allophane and other amorphous constituents in a podzol from Nova Scotia. Can. J. Soil, Sci., 52, 465-475.

GANSSMANN N., 1962. Untersuchungen über den Einfluss der Kieselsaüre auf die Aufnahme von Phosphorsaüre und anderen Nährstoffen. Die Phosphorsaüre, 22 (5/6), 223-241.

GEIS J. W., 1973. Biogenic silica in selected species of decideous angiosperms. Soil Science, 116, 113-129.

GERICKE S., 1962. Kieselsaüre im Thomasphosphat. Die Phosphorsaüre, 22 (5/6), 249-258.

GUILLET B., 1972. Relation entre l'histoire de la végétation et la podzolisation dans les Vosges. Thèse Doct. Etat, Univ. Nancy I, 112 p.

GUILLET B., ROUILLER J., 1974. Fiches de description C. P. B. (inédit).

HALAIS P., PARISH D. H., 1963. Silica and manganese contents of cane leaf sheats in relation to soil and nutrition. Ann. Rep. Mauritius Sugar Industry Res. Inst., 74-76.

HOHNE H., 1963. Der Mineralstoff und Stickstoffgehalt von Waldenpflanzen in Abhängigkeit vom Standort. Arch. für Forstwesen, Berlin, 12, 791-805.

HOHNE H., 1964. Jahreszeitliche Veränderungen des Gewichtes und Elementgehaltes von Fichtennadeln. Arch. für Forstwesen, Berlin, 13, 747-774.

HOLZAPFEL L., ENGEL W., 1954. Der Einfluss organischer Kieselsaüreverbindungen auf das Wachstum von Aspergillus niger und Tristicum. V. Naturforschung, 9b, 602-606.

HOLZAPFEL L., ENGEL W., 1956. Röntgenographischer Nachweis von Quartzkieselsaüre in Weizenpflanzen. Z. Naturforschung, 11b, 226-228.

HOPKINS D. P., 1961. Does silicon matter? Fertil. and Feeding stuffs J., LIX, 467-468.

ISHIBASHI H., AKIYAMA K., 1960. Physiological function of silica in the rice plant. Report III. Bull. Fac. Agr. Yamaguti, 11, 1-8.

IWATA T., BABA I., 1962. Effect of silica upon the adaptability of the rice plant for heavy manuring in relation to photosynthesis. Nippon Sakumotsu Gakkai Kiji, 30, 237-240 (en japonais, résumé en anglais).

JONES L. H. P., HANDRECK K. A., 1967. Silica in soils, plants and animals. Afvances in Agronomy, 107-149.

KAFKAFI U., BAR-YOSEF B., 1969. The effect of $\mathrm{pH}$ on the adsorption and desorption of silica and phosphate on and from kaolinite. Proc. Intern. Clay Conf. Tokyo, Israel Univ. Press, 691-696.

KHAN D. H., ROY A. C., 1964. Growth, P-uptake and fibre all dimensions of jute plant as affected by silicate treatment. Plant and Soil, 20, 331-336.

KUTUZOVA R. S., 1968. Silica transformation during the mineralization of plant residues. Pochvovedeniye, 7, 119-128; Soviet Soil Sc., 7, 970-978.

LANNING F. C., LINKO Y. Y., 1961. Fole of silica in plants. Absorption and deposition of silica by four varieties of Sorghum. J, Agric. Fd. Chem., 6, 463-465.

LAROCHE J., 1967. Localisation de la silice par le micro-analyseur à sonde électronique. C. R. Acad. Sc. Paris, 265D, 1695-1698.

LEWIN J. C., REIMANN B. E. F., 1969. Silicon and plant growth A. Rev. Pl. Physiol., 20, 289-304.

MITSUI S., TAKATOH H., 1963. Nutritional study of silicon in graminaceous crops. Part. 1 and 2 : Soil Sc. Plant Nutr., 9, 7-11, Part 3: The effect of metabolic inhibitors on the silicon uptake of the rice plant. J. Sc. Soil, Tokyo, 33, 449-452.

OKUDA A., TAKAHASHI E., 1964. The role of silicon. Proc. Symp. Mineral Nutrition Rice Plant, 123146.

OVINGTON J. D., 1955. The composition of tree leaves. Forestry, 22-28.

PARRY D. W., SMITHSON F., 1964. Types of opaline silica depositions of grass phytoliths. Ann. Botany London, N. 3, 28 (109), 169-185.

PAVLOV M. B., 1972. Bioelement-Inventur von Buchen und Fichtenbeständen im Solling. Göttingen Bodenkundliche Berichte, 25, $174 \mathrm{p}$.

RAUPACH M., PIPER C. S., 1959. Interactions of silicate and phosphate in a lateritic soil. Austral. J. Agric. Res., 10, 818-831.

ROBERT D., LAROCHE J., GUERVIN C. LECOQ C. SAUVANET A., 1973. Mise en place de la silice dans les cellules épidermiques de la feuille d'une Ptéridophyte : Selaginella kraussiana. II. Etude ultrastructurale. Bull. Museum Hist. Nat., 201, 210-233.

SANGSTER A. G., 1970. Intracellular silica deposition in immature leaves in three species of the Gramineae. Ann. Bot., 34, 245-257. 
SANGSTER A. G., 1970. Intracellular silica deposition in mature and senescent leaves of Sieglingia decumbens L. Ann. Bot., 34, 557-570.

SHIM S. C., YOO S. Y., 1967. Studies on ion absorption of crops by using radioisotopes. III. Effects of silica application of mineral nutrition uptake in rice root. J. Nucl. Sc. Korea, 7, 45-49 (en coréen).

SONI S. L., KAUFMAN P. D., JONES R. A., 1972. Electron micropobe analysis of the distribution of silicon and other elements in rice leaf epidermis. Bot. Gaz., 133 (1), 66-72.

SOUCHIER B., LELONG F., 1972. Comparaison de bilans d'altération sur roches granitiques en zone tempérée et en zone équatoriale. C. R. Acad. Sc. Paris, 274D, 1896-1899.

TOUTAIN F., 1974. Etude écologique de l'humification dans les hêtraies acidiphiles. Thèse Doct. Etat, Univ. Nancy I, $114 \mathrm{p}$.

TWISS P. C., SUESS E., SMITH R. M., 1969. Morphological classificaîion of grass phytoliths. Soil. Sc. Soc. Amer. Proc., 33, 109-115.

VEDY J. C., 1976. Evolution saisonnière des éléments métalliques et de la matière organique dans les pluviolessivats et dans les percolats en liaison avec le mode de pédogenèse. Rapport scient. A. T. P. « Hydrogéologie », CNRS n ${ }^{\circ}$ A 6551635.

VELLY J., 1974. La silice dans la fertilisation du riz à Madagascar. Expérimentations 1969-1973. Document I. R. A. T.

VERMA S. D., RUST R. H., 1969. Observations on opal phytoliths in a soil biosequence in South. eastern Minnesota. Soil Sci. Soc. Proc., 33, 749-751.

VLAMIS J., WILLIAMS D. E., 1967. Manganese and silicon interaction in the gramineae. Plant and Soil, 27, 131-140.

WILDING L. P., DREES L. R., 1971. Biogenic opal in Ohio soils. Soil Sc. Soc. Amer. Proc., 35, 10041010.

WILDING L. P., DREES L. R., 1974. Contributions of forest opal and associated crystalline phases to fine silt and clay fractions of soils. Clays and Clay Minerals, 22, 295-306.

YOSHIDA S., OHNISHI Y., KITAGISHI K., 1962. Soil Sc. Plant Nutr. Tokyo, 8, 30-41, 48-52 et 107-113.

YOSHIDA S., NAVASERO J. A., RAMIREZ E. A., 1969. Effects of silica and nitrogen supply on some leaf characters of the rice plant. Plant and Soil, 31 (1), 48-56. 